\title{
Improvement Measures of 3D Face Recognition Algorithm Based on Features of Facial Contour Curve
}

\author{
ZhaoWeizheng $^{1}$, TangWeiwei ${ }^{2}$ \\ ${ }^{1}$ Zaozhuang Vocational College of Science \& Technology, Shandong, China \\ ${ }^{2}$ Tengzhou second middle school, Shandong, Tengzhou, China
}

Keywords: facial; contour curve; three-dimensional; human face

\begin{abstract}
An improved algorithm based on the feature of facial contour curve is proposed for 3D face recognition. The paper first analyzes the physiological structure of human face, extracts the feature points in the 3D face, and then extracts the face contour line, and combines them to form the feature model to realize the 3D face recognition. The experimental results show that this method can effectively improve the three-dimensional face recognition rate with strong anti-interference ability.
\end{abstract}

\section{Introduction}

The earliest face recognition system has been applied to the identification and authentication of ID cards, passports, and photographs. Currently, it has already been widely used in access control systems and banks. Compared with the identity authentication methods such as iris and fingerprint, face recognition technology has some advantages. It has less user interference and is easier to be accepted. The first face recognition technology was based on two-dimensional features. However, if you want to achieve better recognition results, you need to be based on certain conditions. Due to various factors such as light, expression, and posture, two-dimensional face recognition has some problems in practical applications. The application of three-dimensional face recognition features has strong light, expression, posture and other factors robustness. A three-dimensional face recognition improvement algorithm based on the feature of facial contour curve first extracts the feature points of a human face, then extracts an important contour line of the face, and forms a feature model to recognize a three-dimensional face.

\section{Extract feature points of 3D face}

Face feature points are mostly used in face recognition, organ calibration and face positioning. To complete the recognition of 3D faces, the first step is to collect the feature points that may be used. According to the following principles to select the feature points ${ }^{\text {[11 }}$ : (1)The number should be appropriate, not too much or too little. If the number is too much it will increase the extraction error, and the number is too little to meet the demand; (2)The feature points need to react to the overall geometry of the human face, and they cannot affect facial expressions. (3)The selected feature points should be easily located on the computer.

\subsection{Using differential algorithm to identify local features}

The three-dimensional surface data is organized according to layers. If we can obtain the three-dimensional surfaces, then the relevant organ contour edge information can be obtained according to the variation of the center axis distance. First, calculate the centroid of each layer and use it as the center point of the layer, and calculate the radius of each discrete point to the centroid of the layer, use radial radius to calculate the difference between the discrete points of each parameter surface and the surrounding four neighborhoods. After calculating a complete three-dimensional surface difference, you can obtain a facial difference map ${ }^{\mathbf{2} 2}$. See Figure 1 below. 

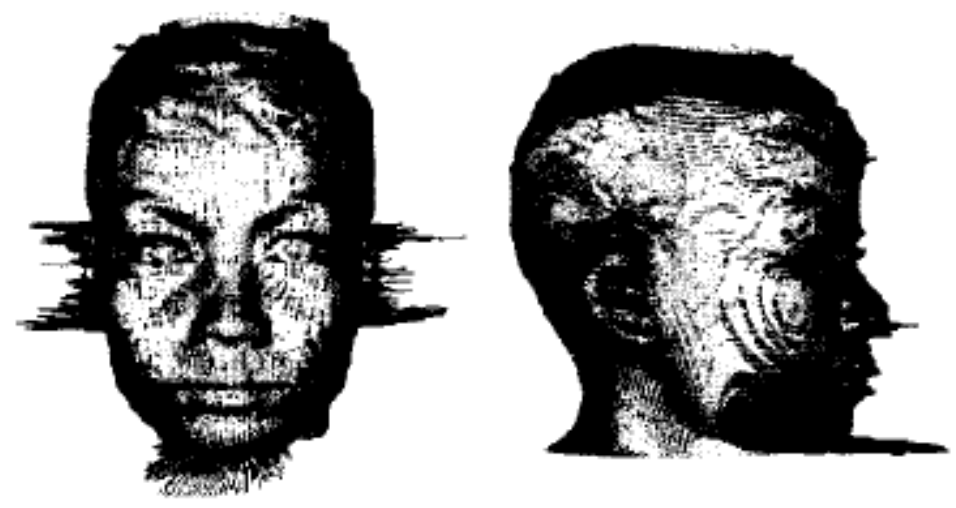

Figure 1 Front and side difference images

The centroid of the layer expressed as formula (1):

$$
\mathrm{O}_{\mathrm{c}, \mathrm{i}}(\mathrm{x}, \mathrm{y}, \mathrm{z})=\sum_{\mathrm{j}=0}^{\text {Current layer points numbers }} \mathrm{P}_{\mathrm{i}, \mathrm{j}}(\mathrm{x}, \mathrm{y}, \mathrm{z})
$$

In formula (1), i represents the current layer number, and $\mathrm{j}$ represents the number of points on the current layer.

The radial radius of points $i$ and $j$ in formula (1) is expressed as:

$$
\mathrm{R}_{\mathrm{i}, \mathrm{j}}=\sqrt{\left(\mathrm{P}_{\mathrm{i}, \mathrm{j}}(\mathrm{x})-\mathrm{O}_{\mathrm{c}, \mathrm{i}}(\mathrm{x})\right)^{2}+\left(\mathrm{P}_{\mathrm{i}, \mathrm{j}}(\mathrm{y})-\mathrm{O}_{\mathrm{c}, \mathrm{i}}(\mathrm{y})\right)^{2}+\left(\mathrm{P}_{\mathrm{i}, \mathrm{j}}(\mathrm{z})-\mathrm{O}_{\mathrm{c}, \mathrm{i}}(\mathrm{z})\right)^{2}}
$$

The vertical forward difference and the horizontal forward difference are shown in formula (3) and formula (4) respectively:

$$
\begin{gathered}
v-\text { differ }_{i . j}=R_{i+1, j}-R_{i, j} \\
h-\text { differ }_{i . j}=R_{i, j+1}-R_{i, j}
\end{gathered}
$$

Combining formulas (3) and (4) to obtain the forward mixture difference, as shown in formula (5):

$$
\operatorname{differ}_{\mathrm{i}, \mathrm{j}}=\frac{\mid v-\text { differ }_{i, j}|+| h-\text { differ }_{i . j} \mid}{2}=\frac{\left|R_{i+1, j}-R_{i, j}\right|+\left|R_{i, j_{+1}}-R_{i, j}\right|}{2}
$$

\subsection{Constructing the edge area of the organ and perform feature positioning}

Organize the edge points of the same organ so that they can form an area together. In this study, the regional growth seed algorithm was used to find the contour region of the organ. Since the overall contour of an organ is likely to be divided into different pieces that are stored in the list of regions, it is necessary to merge these segmented blocks before calculating the features. The mouth and eyes are segmented according to the template so that the boundary parameters of the organs are fitted to them. For the mouth, we used the vertical direction of the region to obtain the mean value, and obtained a "middle-axis point set" from the two corners of the mouth to the middle, and divided it into the upper, middle and lower three parts. We also obtained the "middle axis point set" from two corners of the eye to the middle of the eye in this way.

\section{The extraction of optimal profile curves}

In pattern recognition, the extracted facial curve features have a direct effect on the recognition result. For example, when extracting facial contour features, equal interval extraction is used. Although the operation is relatively simple and easy to implement, the contour curve extracted by this method is not necessarily the most representative, it could easily cause the loss of important facial features. Moreover, for some contour curves with similar face information, the extracted part 
of the contour curve recognition function may be relatively small. Therefore, the most representative facial contour curve needs to be extracted.

In the process of face recognition, the face information is fully expressed using less contour lines as much as possible, and the recognition rate can be improved. The fuzzy clustering method is used to cluster multiple contour curves and divide similar clusters. When identifying, a feature contour line is selected from each contour line to ensure that the contour lines represent unique feature information, which is conducive to achieving the best feature ${ }^{\mathbf{3} \mathbf{I}}$.

As shown in Fig. 2, the face depth gallery is used as a three-dimensional face when performing recognition experiments. The situation shown in Fig. 2(a) is a front view of a person and 14 images acquired based on different expressions and lighting conditions; The situation shown in FIG. 2(b) is a graph obtained when the human face is viewed from the side. In order to ensure that important facial feature information is not lost, the contour lines selected from these face depth maps need to be kept within the $[1,255]$ depth value interval, and 26 contour lines are extracted at an interval of 10 to represent the more representative face feature information. as shown in Figure 3.

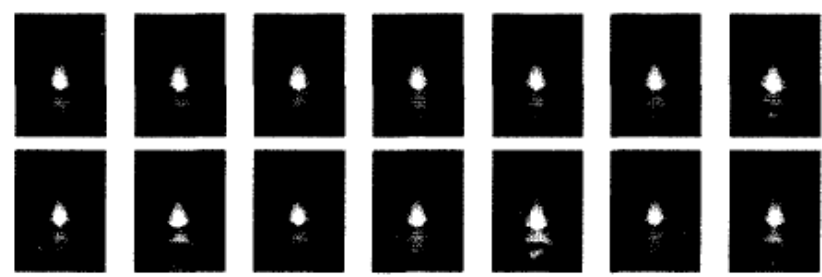

(a) Different images observed in front by the same person

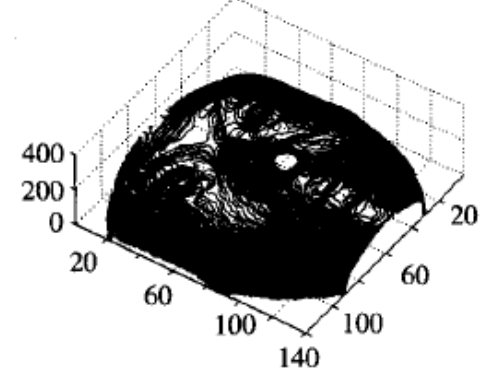

(b) Side view depth map

Figure 2 Face Depth Gallery
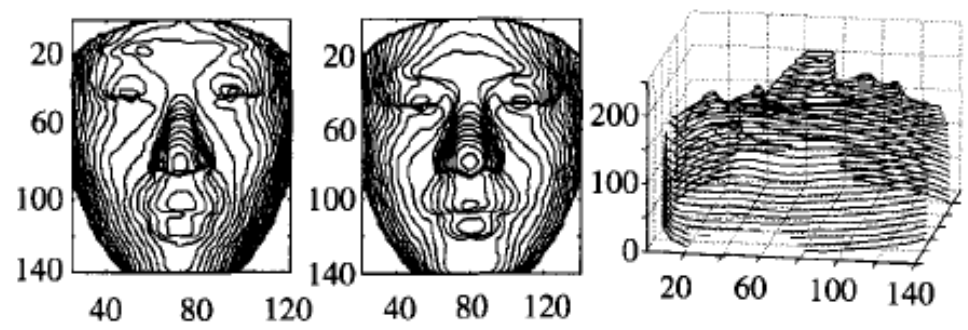

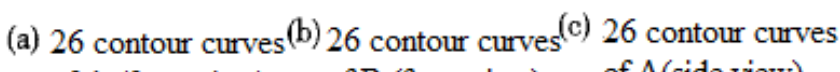
of A (front view) of B (front view) of A(side view)

Figure 3 extracted outlines

The extracted 26 contours can be clustered according to the fuzzy relation clustering method to obtain the most representative contour curve. If the object to be classified is set to $n$, then $\mathrm{n}=26$. Assuming that the features of each contour line contain s dimensions, the assumption is: $\mathrm{X}_{1 \mathrm{k}}$, $\mathrm{X}_{2 \mathrm{k}}, \ldots \mathrm{X}_{\mathrm{nk}}, \mathrm{k}=1,2, \ldots$ s. The clustering algorithm calculates steps are as follows: (1)Data standardization. Calculating the feature variance and mean of each dimension:

$$
\mathrm{S}_{\mathrm{k}}^{2}=\frac{1}{\mathrm{n}} \sum_{\mathrm{i}=1}^{\mathrm{n}}\left(\mathrm{X}_{\mathrm{ik}}-\mathrm{X}_{\mathrm{k}}\right)^{2}
$$




$$
\mathrm{X}_{\mathrm{k}}=\frac{1}{\mathrm{n}} \sum_{\mathrm{i}=1}^{\mathrm{n}} \mathrm{X}_{\mathrm{ik}}
$$

(2)D ata extremum standardization. Ensuring that all data are in the $[0,1]$ interval:

$$
\mathrm{X}_{\mathrm{ik}}^{\prime \prime}=\frac{\mathrm{X}_{\mathrm{ik}}^{\prime}-\mathrm{X}_{\mathrm{kmin}}^{\prime}}{\mathrm{X}_{\mathrm{kmax}}^{\prime}-\mathrm{X}_{k \min }^{\prime}}
$$

In formula (8), $X_{\mathrm{ik}}^{\prime \prime}=\frac{X_{\mathrm{ik}}-\mathrm{X}_{\mathrm{k}}}{\mathrm{S}_{\mathrm{k}}}, \mathrm{X}_{\mathrm{kmin}}^{\prime}$ and $\mathrm{X}_{\mathrm{kmax}}^{\prime}$ represented the minimum and maximum value respectively, and $\mathrm{i}=1,2, \ldots, \mathrm{n}$.

(3) Making full use of the classification object set to define the fuzzy similarity relation matrix R. Fuzzy similarity relation matrix $R$ is obtained by calculation. $R=\left[r_{i j}\right]$, As formula (9):

$$
r_{i j}=\frac{\sum_{k=1}^{m} X_{i k} X_{j k}}{\sqrt{\sum_{k=1}^{m} X_{i k}^{2} \sum_{k=1}^{m} X_{j k}^{2}}}
$$

(4)We choose the Warshall algorithm for the construction of the transitive closure of the fuzzy relation matrix $\mathrm{R}$, and then get the fuzzy equivalence relation matrix $\mathrm{R}^{*}$ by calculation. Based on the requirement that classification relations need to be transitivity, symmetry and reflexivity, fuzzy similarity relation $\mathrm{R}$ is symmetry and reflexivity, but it cannot be transitivity, so it needs to be transformed into fuzzy equivalence relation ${ }^{\mathbf{1}}$. By using the Warshall algorithm, the matrix $(\mathrm{L}+1)$ is constructed by recursive method. The calculation results are as follows: when $\mathrm{k}=\mathrm{L}$, the result of the calculation is a fuzzy equivalent relationship matrix $\mathrm{R}^{*}$.

The optimal division result is to first consider the basic characteristics and ensure that the curves between classes are as different as possible. The main basis for rational division is the structure of data sets, and the quality of clustering is evaluated. If it is assumed that the original sample curve set is $\mathrm{S}=\left\{\mathrm{X}_{1}, \mathrm{X}_{2}, \ldots, \mathrm{X}_{\mathrm{n}}\right\}$, after clustering, it is divided into $\mathrm{m}$ classes, then $\mathrm{C}=\left\{\mathrm{C}_{1}, \mathrm{C}_{2}, \ldots, \mathrm{C}_{\mathrm{m}}\right\}(\mathrm{m} \leq \mathrm{n})$. If $\mathrm{C}$ can meet the conditions of clustering partition, the cluster validity indicator function can be defined, such as formula (10) and formula (11):

$$
\begin{aligned}
& \mathrm{S}_{\mathrm{n}}\left(\mathrm{C}^{\mathrm{m}}\right)=\sum_{\mathrm{i}=1}^{\mathrm{m}} \sum_{\mathrm{X}_{\mathrm{a}},} \mathrm{X}_{\mathrm{b}} \in \mathrm{C}_{\mathrm{i}} \mathrm{X}_{\mathrm{a}} \mathrm{X}_{\mathrm{b}} \\
& S_{j}\left(C^{m}\right)=\sum_{i=1}^{m} \sum_{j=1, j \neq i}^{m} \frac{1}{\left|C_{i} \| C_{j}\right|_{X_{a} \in C_{i}}, X_{b} \in C_{j}} R X_{a} X_{b}
\end{aligned}
$$

The $\mathrm{RX}_{\mathrm{a}} \mathrm{X}_{\mathrm{b}}$ in formulas (10) and (11) represent the cosine similarity values of $\mathrm{Xa}$ and $\mathrm{Xb}$. Sn indicates internal similarity. $\left|C_{i}\right|$ indicates the number of curves to be assigned to this class. $\mathrm{Sj}$ indicates similarity between classes. To ensure that $\mathrm{Sn}$ is metrically consistent with $\mathrm{Sj}$, the evaluation function is defines as:

$$
\mathrm{W}\left(\mathrm{C}^{\mathrm{m}}\right)=\mathrm{S}_{\mathrm{n}}\left(\mathrm{C}^{\mathrm{m}}\right)+\mathrm{S}_{\mathrm{j}}\left(\mathrm{C}^{\mathrm{m}}\right)
$$

If $\mathrm{W}\left(\mathrm{C}^{\mathrm{m}}\right)$ in equation (12) is the smallest, the optimal clustering amount can be obtained. According to experiments, when $\mathrm{m}=7$, it has a poor recognition rate, and when $\mathrm{m}=8$, it has a good clustering effect. We selected 8 contour lines, as shown in Figure 4. (a) and (c) in FIG. 4 are the original 26 contour lines, and (b) and (d) represent the eight contour lines after the extraction. The recognition of $3 \mathrm{D}$ face can be classified by contour line. 


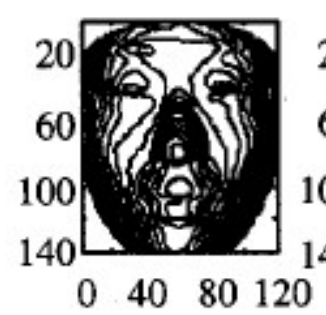

26 contour curve

(a) of human $\mathrm{A}$

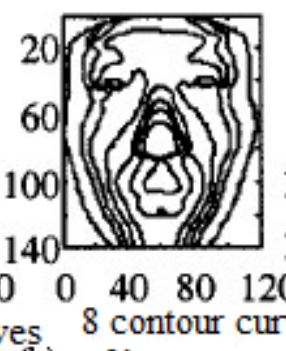

(b) of human $\mathrm{A}$

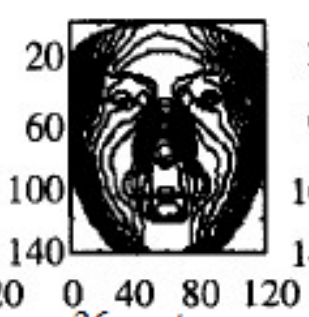

(c) of human B

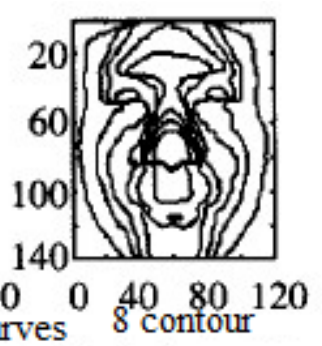

(d) curves of human B

Figure 4: Contour extraction of human A and human B faces respectively

The comparison of the contour lines obtained by adopting the equal interval sampling and adopting the fuzzy clustering method is shown in Figure 5.

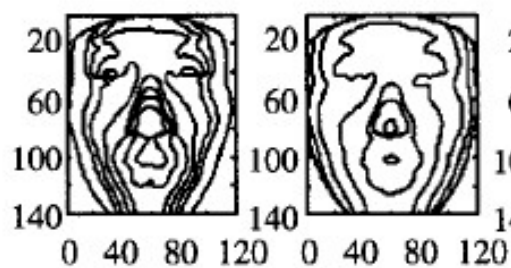

(a) 8 contour cur human $\mathrm{A}$ (b) 8 contour curves of
the equal spacing
of human $\mathrm{A}$

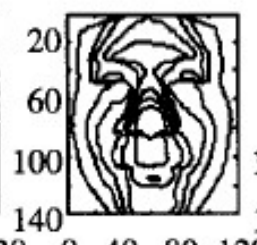

4080120

curves of human B

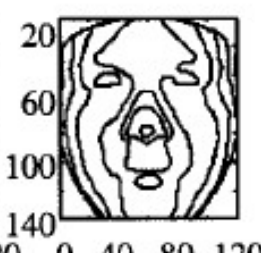

$\begin{array}{lllll}0 & 40 & 80 & 120\end{array}$

(d) 8 contour curves

(d) of the equal spacing of human B

Figure 5 Comparison of contour lines extracted by equal interval sampling and fuzzy clustering

It can be seen from Figure 5 that the contour lines extracted by the fuzzy clustering fee method are relatively concentrated in the special feature information area where face is rich, and there are relatively large shape differences. Because the feature of the contour curve belongs to the depth value extraction $^{\mathbf{5 1}}$ ], it has great difference, so it can represent more comprehensive information. In different faces, if there is a large difference in the contour of the same depth value, the similarity of the face shape is smaller. The extraction contour is sampled and extracted in an equally spaced manner. The extracted contour lines are mostly concentrated on the edges of the human face. Therefore, the lack of face contour feature information will generate similar partial contour lines and the comprehensive information cannot be displayed ${ }^{\mathbf{6} \mathbf{}}$. If you increase the number of contour lines, although you can enrich the feature information further, the corresponding calculation complexity will also increase. Therefore, the use of fuzzy clustering method can better obtain important contour features ${ }^{\mathbf{7 1}}$.

\section{Test and result analysis}

22 faces were tested, and 24 sets of 3D data of human head were obtained by 3D scanning and CT reconstruction. According to the above methods, feature points and important contour lines were extracted. Because feature point and outline extraction will produce certain errors, the feature points and the important contours are matched first. The result of the experiment is compared with the method of recognizing the three-dimensional face by the use of separate contour lines. The results show that the recognition of the incoming three-dimensional face using the combination of feature points and important contours can accurately identify 22 faces, and the recognition speed is $\leq 0.8 \mathrm{~s}^{\mathrm{r}}{ }^{\mathrm{p} 1}$.

\section{Conclusions}

Through this study, the problem of two-dimensional face recognition can be solved by using three-dimensional samples to identify faces and by the way of extracting feature points and 
important contour lines. It has been proved by experiments that the face can be accurately identified. The method is feasibility.

\section{References}

[1] Lin Kezheng, Xu Songting, Li Xin, Wu Di. Multi-information fusion face recognition using key feature points[J]. Journal of Harbin University of Science and Technology,2017,22(03):19-24.

[2] Wang Jian, Gao Yuan, Qin Pinle, Wang Lifang. Three-dimensional face recognition based on improved LBP algorithm[J]. Computer Engineering and Design,2016,37(12):3366-3370.

[3] Liu Shumu, Yang Jian, Chen Yue. Conformal Feature Combined with Improved Differential Evolution Algorithm for 3D Face Recognition[J]. Application Research of Computers, 2016,33(06):1898-1902.

[4] Wang Siteng, Tang Xusheng, Chen Dan. Application of neural network with feature fusion in 3D face recognition[J]. Machinery Manufacturing \& Automation,2015,44(04):126-131.

[5] Wang Xiaobin, Feng Luqiao, Yang Yuanjing. Three-dimensional face matching method based on simulated annealing algorithm[J]. Journal of University of Electronic Science and Technology of China,2015,44(04):584-588.

[6] Tao Lijun, Zhang Xijing. Research on 3D Face Recognition Based on Improved LBP Operator and Sparse Representation[J]. Modern computer,2013(14):23-26. 